\title{
HENSELIZATION OF A RING WLTH RESPECT TO AN IDEAL
}

\author{
BY \\ SILVIO GRECO( $\left.{ }^{(}\right)$
}

Introduction. The concept of henselization of a local ring, introduced by Nagata ([11], [12]) in 1953-54, was later extended to the pairs $(A, \mathfrak{m}),(A$ a commutative ring, $\mathfrak{m}$ an ideal) by J. P. Lafon, who in 1963 proved the existence theorem [10, Theorem 1].

Many properties of the henselization of a local ring are known, mainly due to Nagata: for example the henselization of a local $\operatorname{ring} A$ is a local ring $B$ which is faithfully flat over $A$, has the same completion as $A$, and is noetherian (normal, reduced) if and only if so is $A[13$, Chapter VII]; $[9,18.6]$.

The aim of this paper is to investigate the nonlocal situation, trying to extend to the henselization of a pair some results which are known for local rings.

This paper can be divided into three parts. In the first one ( $\$ 1$ to 3 ) we construct, given a pair $(A, \mathfrak{m})$, a direct set of pairs (the " $N$-extensions"), whose direct limit is the henselization of $(A, \mathfrak{m})$, as we show in the second part $(\$ \$ 4,5)$. The techniques used here were sketched in [5], and extend similar arguments used by Nagata and Lafon in the study of the local case [10, $\$ 4]$ and [13, Chapter VII]. In the last part ( $\$ 6$ to 9) we use the properties of the $N$-extensions, in order to "delocalize" several theorems on the henselization of a local ring.

$\S 1$ is introductory: after some remarks on the category of pairs $(A, \mathfrak{m})(A$ commutative ring, $\mathfrak{m}$ ideal), we define $H$-pairs (Hensel pairs) (Definition 1.1) and $N$-pairs (Definition 1.3), which are, in appearance, a wider class than $H$-pairs. Then we define the $\mathrm{N}$-closure and $\mathrm{H}$-closure of a pair, as solutions of similar universal problems (Definition 1.7): it will turn out later that $H$-closure and $N$-closure coincide (Theorem 5.10), and that $H$-pairs and $N$-pairs are the same thing (Theorem 5.11).

In $\$ 2$ we begin to construct the $N$-closure of a pair, by defining the " $N$-extensions" of a given pair, (Definition 2.2) and by giving their elementary properties (Proposition 6.2). In $\S 3$ we show that the $N$-extensions of $(A, \mathfrak{m})$ form a direct set (Proposition 3.3), and that their direct limit $\left({ }^{n} A,{ }^{n} \mathfrak{m}\right)$ is the $N$-closure of $(A, \mathfrak{m})$ (Theorem 3.5).

At this point our problem is to show that $\left({ }^{n} A,{ }^{n} \mathfrak{m}\right)$ is an $H$-pair, whence it coincides with the henselization of $(A, \mathfrak{m})$. Thus, in $\$ 4$, we study more closely the $N$-pairs (Proposition 4.10) in order to show an $N$-pair $(A, \mathfrak{m})$ with $A$ regular is an

Received by the editors November 25, 1968 .

(1) This research was done when the author was a member of the 32nd research group of the C.N.R. 
$H$-pair (Corollary 4.11). In the following section we show, by using properties of completions, that if $A$ is regular so is ${ }^{n} A$, whence, by the above result $\left({ }^{n} A,{ }^{n} \mathfrak{m}\right)$ is an $H$-pair whenever $A$ is regular (Proposition 5.5). The general problem can be reduced to this one (Theorem 5.10), and so we have that $\left({ }^{n} A,{ }^{n} \mathfrak{m}\right)$ is the henselization of $(A, \mathfrak{m})$.

This concludes $\S 5$. In the remaining sections we use some nice properties of the $N$-extensions in order to investigate henselization more closely. In $\S 6$ we show that if $(B, \mathfrak{n})$ is the henselization of $(A, \mathfrak{m})$, then: (a) $B$ is flat over $A$ and is faithfully flat over $A$ if and only if $m \subset \operatorname{rad} A$ (Theorem 6.5), and (b) if $A$ is noetherian so is $B$; and if $B$ is noetherian and $\mathfrak{m} \subset \operatorname{rad} A$ then $A$ is noetherian (Theorem 6.8, Corollary 6.9).

$\$ 7$ is devoted to local properties. It is shown that some local problems can be reduced to henselization of local rings (Theorem 7.4, Corollary 7.5): in particular properties as locally normal, regular, Cohen-Macaulay, $R_{k}, S_{k}$ are preserved by henselization (Corollaries 7.6, 7.7); and the henselization of a locally unibranch reduced ring (Definition 7.8) is always locally a domain (Corollary 7.9).

In $\S 8$ we deal with reducedness, by the aid of Theorem 7.4. The main result is Theorem 8.7: if $(B, \mathfrak{n})$ is the henselization of $(A, \mathfrak{m})$, then $\left(B_{\text {red }}, \mathfrak{n} B_{\text {red }}\right)$ is the henselization of $\left(A_{\text {red }}, \mathfrak{m} A_{\text {red }}\right)$. In particular if $A$ is reduced so is $B$.

Finally, in $\S 9$, we give criteria for the henselization of a noetherian ring to be a domain (Theorem 9.3), a normal domain (Theorem 9.5), a ring with irreducible spectrum (Theorem 9.7) and a UFD (Theorem 9.11).

Many of the results of $\$ \S 6$ to 9 are similar to properties of $m$-adic completions of noetherian rings. However henselization usually behaves much better than completion, also without noetherian assumptions, as e.g. with regards to flatness, normality and reducedness. By this it is reasonable to hope that henselization of a pair may have some geometric applications, as is already happening for local henselization (e.g. [9], §18).

1. In this section we fix some notations and definitions we will use in the rest of the paper.

All rings and algebras considered are supposed to be commutative with 1, ring homomorphisms map 1 into 1 , and algebras and modules are unitary.

We will be dealing with pairs $(A, \mathfrak{m})$, where $A$ is a ring and $\mathfrak{m}$ an ideal of $A$. Notations as $(A, \mathfrak{m})(B, \mathfrak{n}), \ldots$ will always indicate such pairs.

A morphism $\phi:(A, \mathfrak{m}) \rightarrow(B, \mathfrak{n})$ is a ring homomorphism $\phi: A \rightarrow B$ such that $\phi^{-1}(\mathfrak{n})=\mathfrak{m}$. (This generalizes the definition of a local homomorphism of local rings.)

It is clear that the family of pairs with the morphisms just defined form a category $\mathscr{P}$, the category of pairs. (For more details on the category $\mathscr{P}$ see [10].)

If $(A, \mathfrak{m})$ is a pair, we will denote by $(\hat{A}, \hat{\mathfrak{m}})$ its completion; by this we mean that $\hat{A}, \hat{\mathfrak{m}}$ are the completions of $A$ and $\mathfrak{m}$ with respect to the $\mathfrak{m}$-topology. We will write, sometimes, $(A, \mathfrak{m})^{\wedge}$ in place of $(\hat{A}, \hat{\mathfrak{m}})$. 
The canonical homorphism $A \rightarrow \hat{A}$ is actually a morphism of pairs $(A, \mathfrak{m})$ $\rightarrow(\hat{A}, \hat{\mathfrak{m}})$.

A pair $(A, \mathfrak{m})$ is complete if it coincides with its completion, i.e. if

$$
A=\operatorname{proj} \lim A / \mathfrak{m}^{n}=\hat{A}
$$

(For details on completions see e.g. [3, Chapter III].)

As Hensel's lemma shows [3, p. 84, Theorem 1], complete pairs are a particular class of $H$-pairs (or Hensel pairs) which are defined as follows (cf. [10]):

Definition 1.1. $(A, \mathfrak{m})$ is an $H$-pair if the following conditions are satisfied:

(i) $\mathfrak{m} \subset \operatorname{rad}(A)$.

(ii) Hensel's lemma holds for every monic polynomial in $A[X]$.

There are several conditions which are equivalent to Definitions 1.1 (see [10], [7], and Theorem 5.11).

Now we are going to define $N$-polynomials and $N$-pairs, which will play an important role in our construction of henselization.

Definition 1.2. Let $(A, \mathfrak{m})$ be a pair. A monic polynomial $a_{0}+a_{1} X+\cdots+X^{n}$ is called an $N$-polynomial over $(A, \mathfrak{m})$ if $a_{0} \in \mathfrak{m}$, and $a_{1}$ is a unit $\bmod \mathfrak{m}$.

DeFinition 1.3. $(A, \mathfrak{m})$ is an $N$-pair if

(i) $\mathfrak{m} \subset \operatorname{rad} A$.

(ii) every $N$-polynomial over $(A, \mathfrak{m})$ has a root in $\mathfrak{m}$.

By a straightforward computation we have:

Lemma 1.4. Every H-pair (Definition 1.1) is an N-pair.

We will prove later (Theorem 5.11) that the converse of Lemma 1.4 is true. But in order to do that we have to study $N$-pairs in more detail. We begin by observing that the root of the $N$-polynomials in Definition 1.3 is unique:

Lemma 1.5. Let $F(X)$ be an $N$-polynomial over $(A, \mathfrak{m})$. If $\mathfrak{m} \subset \operatorname{rad} A, F(X)$ has at most one root in $\mathrm{m}$.

Proof. Suppose $x, y \in \mathfrak{m}$ are such that $F(x)=F(y)=0$. Then we have, for suitable monic polynomials $G(X), H(X) \in A[X]$ :

$$
(X-x) G(X)=(X-y) H(X)=F(X) .
$$

Putting $X=x$ in (1) we get

$$
(x-y) H(x)=0
$$

and to conclude the proof we show that $H(x)$ is a unit.

Taking derivatives in (1) we get

$$
F^{\prime}(X)=(X-y) H^{\prime}(X)+H(X)
$$

and, putting $X=x$,

$$
F^{\prime}(x)=(x-y) H^{\prime}(x)+H(x)
$$


Since $\mathfrak{m} \subset \operatorname{rad} A$ and $F(X)$ is an $N$-polynomial over $(A, \mathfrak{m})$ it is easy to see that $F^{\prime}(x)$ is a unit. But $x-y \in \mathfrak{m} \subset \operatorname{rad} A$, and hence by (3) one sees immediately that $H(x)$ is a unit. Thus $x=y$ by (2), and the proof is complete.

COROllary 1.6. Let $(A, \mathfrak{m})$ be an $N$-pair. Then every $N$-polynomial over $(A, \mathfrak{m})$ has one and only one root in $\mathfrak{m}$. This is true, in particular, if $(A, \mathfrak{m})$ is an H-pair (Lemma 1.4).

As we wrote in the Introduction, the aim of this paper is to study the henselization (or $H$-closure).

Definition 1.7. Let $(A, \mathfrak{m})$ be a pair. An $H$-pair (resp. an $N$-pair) $(B, \mathfrak{n})$, together with a morphism $\phi:(A, \mathfrak{m}) \rightarrow(B, \mathfrak{n})$ is an $H$-closure (resp. an $N$-closure) of $(A, \mathfrak{m})$ if for any $H$-pair (resp. $N$-pair) $\left(B^{\prime}, \mathfrak{n}^{\prime}\right)$ and any morphism $\psi:(A, \mathfrak{m})$ $\rightarrow\left(B^{\prime}, \mathfrak{n}^{\prime}\right)$, there is a unique morphism $\psi^{\prime}:(B, \mathfrak{n}) \rightarrow\left(B^{\prime}, \mathfrak{n}^{\prime}\right)$ such that the following diagram is commutative:

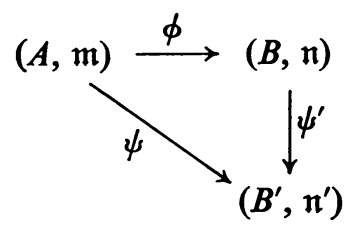

It is clear that $H$-closure and $N$-closure are unique up to canonical isomorphisms. The existence of the $H$-closure was proved by J. P. Lafon [10, Theorem 1]. Here we give an explicit construction of the $N$-closure (Theorem 3.5), and we will prove that it coincides with the $H$-closure (Theorem 5.10). This construction, beside giving a new proof of Lafon's theorem, allows us to prove several properties of henselization (see $\$ 6$ to 9).

2. This section and the one which follows are devoted to the construction of the $N$-closure of a pair.

The ideas of this construction are inspired by similar constructions for local henselization, and were sketched in our note [5]. Here we give a more complete and general treatment.

In this section we begin to develop some preliminary remarks on strict morphisms, and define a class of such morphisms (the $N$-extensions) which will play the main role in our construction.

Definition 2.1. A morphism $\phi:(A, \mathfrak{m}) \rightarrow(B, \mathfrak{n})$ is strict if

(i) $\mathfrak{n}=\mathfrak{m} B$ (we write $\mathfrak{m} B$ in place of $\phi(\mathfrak{m}) B$ ).

(ii) $\phi$ induces an isomorphism $A / \mathfrak{m} \rightarrow B / \mathfrak{n} B$.

Definition 2.2. Let $(A, \mathfrak{m})$ be a pair and $F(X)$ an $N$-polynomial over $(A, \mathfrak{m})$ (Definition 1.2). Let $A[x]=A[X] /(F(X)), S=1+(\mathfrak{m}, x) A[x]$ and $B=S^{-1} A[x]$. We say that $(B, \mathfrak{m} B)$ is a simple $N$-extension of $(A, \mathfrak{m})$.

An $N$-extension of $(A, \mathfrak{m})$ is a pair $(B, \mathfrak{n})$ obtained from $(A, \mathfrak{m})$ by a finite number of simple $N$-extensions. 
We are now going to show that if $(B, \mathfrak{n})$ is an $N$-extension of $(A, \mathfrak{m})$ there is a canonical morphism $(A, \mathfrak{m}) \rightarrow(B, \mathfrak{n})$, and this morphism is strict (Proposition 2.6).

Lemma 2.3. Let $(B, \mathfrak{n})$ be a simple $N$-extension of $(A, \mathfrak{m})$ and let $B=S^{-1} A[x]$ as in Definition 2.2. Then $x \in \mathfrak{n}$.

Proof. By definition there is a relation $a_{0}+a_{1} x+\cdots+x^{n}=0$ with $a_{0}, \ldots, a_{n-1}$ $\in A, a_{0} \in \mathfrak{m}$ and $a_{1}$ invertible $\bmod \mathfrak{m}$. Therefore $x\left(a_{1}+a_{2} x+\cdots+x^{n-1}\right) \in \mathfrak{m} B=\mathfrak{n}$. By construction of $B$ we have $(\mathfrak{m}, x) B \subset \operatorname{rad} B$, whence $a_{1}+a_{2} x+\cdots+x^{n-1}$ is a unit in $B$ and $x \in \mathfrak{n}$.

Lemma 2.4. Let $(B, \mathfrak{n})$ be a simple $N$-extension of $(A, \mathfrak{m})$, and let $\phi: A \rightarrow B$ be the canonical homomorphism. Then $\phi^{-1}(\mathfrak{n})=\mathfrak{m}$ and $\phi:(A, \mathfrak{m}) \rightarrow(B, \mathfrak{n})$ is a morphism of pairs.

Proof. We use again the notations as in Definition 2.2. Let $\psi: A[x] \rightarrow B$ be the canonical homomorphism. Then it is easy to see that $\psi^{-1}(\mathfrak{m}, x) B=(\mathfrak{m}, x) A[x]$. But $x \in \mathfrak{n}$ by Lemma 2.3, whence $(\mathfrak{m}, x) B=\mathfrak{m} B=\mathfrak{n}$ and $\psi^{-1}(\mathfrak{n})=(\mathfrak{m}, x) A[x]$.

Observe now that $A[x]$ is a free $A$-module (a basis is $1, x, \ldots, x^{n-1}$ ); by this it is easy to show that $(\mathfrak{m}, x) \cap A=\mathfrak{m}$, and the conclusion follows immediately.

LemMA 2.5. Let $(B, \mathfrak{n})$ be a simple $N$-extension of $(A, \mathfrak{m})$, and let $\phi:(A, \mathfrak{m})$ $\rightarrow(B, \mathfrak{n})$ be the canonical morphism (Lemma 2.4). Then $\phi$ is strict (Definition 2.1).

Proof. By Definition 2.2 we have $\mathfrak{n}=\mathfrak{m} B$. Therefore it remains to show that $\phi$ induces an isomorphism $\phi: A / \mathfrak{m} \rightarrow B / \mathfrak{n}$ (Definition 2.1(ii)), and since $\phi^{-1}(\mathfrak{n})=\mathfrak{m}$ (Lemma 2.4), it is sufficient to show that $\phi$ is onto.

Write $B=S^{-1} A[x]$ as in Definition 2.2. Then if $\alpha \in B$ we have

$$
\alpha=\frac{b_{0}+b_{1} x+\cdots+b_{n-1} x^{n-1}}{1+m_{0}+m_{1} x+\cdots+m_{n-1} x^{n-1}}
$$

where $b_{0}, \ldots, b_{n-1}, m_{0}, \ldots, m_{n-1} \in A$ and $\mathfrak{m}_{0} \in \mathfrak{m}$. Let $\bar{\alpha}$ be the image of $\alpha$ in $B / \mathfrak{n}$. Since $x \in \mathfrak{n}$ (Lemma 2.3), we have $\bar{\alpha}=\bar{b}_{0}$, whence the conclusion.

Proposition 2.6. Let $(B, \mathfrak{n})$ be an $N$-extension of $(A, \mathfrak{m})$. Then there is a canonical morphism $\phi:(A, \mathfrak{m}) \rightarrow(B, \mathfrak{n})$ which is strict (Definition 2.1).

Proof. By Lemma 2.3 it is clear that $\phi$ exists, and since a composition of strict morphisms is clearly a strict morphism, the conclusion follows by Lemma 2.5 and the definition of $\mathrm{N}$-extension.

3. In this section we will construct the $N$-closure of a pair (Definition 1.7). In order to do this, we show that the set of all $N$-extensions of a given pair is a direct set (Proposition 3.3): its direct limit will be the $N$-closure (Theorem 3.5).

We need several lemmas.

LEMma 3.1. Let $\left(A^{\prime}, \mathfrak{m}^{\prime}\right)$ be an $N$-extension of $(A, \mathfrak{m})$ and let $(B, \mathfrak{n})$ be a pair with $\mathfrak{n} \subset \operatorname{rad} B$. Let $\phi:(A, \mathfrak{m}) \rightarrow\left(A^{\prime}, \mathfrak{m}^{\prime}\right)$ be the canonical morphism. 
Then for any morphism $\psi:(A, \mathfrak{m}) \rightarrow(B, \mathfrak{n})$ there is at most one morphism $\psi^{\prime}:\left(A^{\prime}, \mathfrak{m}^{\prime}\right) \rightarrow(B, \mathfrak{n})$ such that $\psi^{\prime} \circ \phi=\psi$.

Proof. It is clear that we may suppose $\left(A^{\prime}, \mathfrak{m}^{\prime}\right)$ is a simple $N$-extension. Then there is an $N$-polynomial $F(X)$ over $(A, \mathfrak{m})$ such that $A^{\prime}=S^{-1} A[x]$ where $A[x]$ $=A[X] /(F(X))$, and $S=1+(\mathfrak{m}, x) A[x]$. Moreover $\mathfrak{m}^{\prime}=\mathfrak{m} A^{\prime}$ (Definition 2.2).

Let now $\psi_{i}:\left(A^{\prime}, \mathfrak{m}^{\prime}\right) \rightarrow(B, \mathfrak{n}), i=1,2$, be two morphisms such that $\psi_{i} \circ \phi=\psi$ $(i=1,2)$. Since $x \in \mathfrak{m}^{\prime}$ (Lemma 2.3) we have $y_{i}=\psi_{i}(x) \in \mathfrak{n}$.

Let $G(X)=\phi(F(X))$. Then $G(X)$ is an $N$-polynomial over $(B, \mathfrak{n})$, and $G\left(y_{1}\right)=0$ $=G\left(y_{2}\right)$. But $y_{i} \in \mathfrak{n} \subset \operatorname{rad} B$, whence $y_{1}=y_{2}$ (Lemma 1.5), and the conclusion follows easily.

Corollary 3.2. Let $\left(A^{\prime}, \mathfrak{m}^{\prime}\right)\left(A^{\prime \prime}, \mathfrak{m}^{\prime \prime}\right)$ be two $N$-extensions of $(A, \mathfrak{m})$. Then there is at most one morphism $\psi:\left(A^{\prime}, \mathfrak{m}^{\prime}\right) \rightarrow\left(A^{\prime \prime}, \mathfrak{m}^{\prime \prime}\right)$ such that the following diagram is commutative:

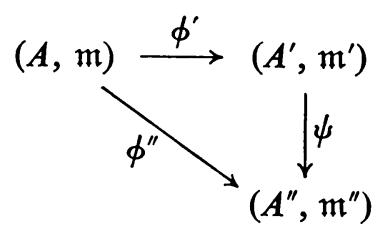

Proof. Since by construction $\mathfrak{m}^{\prime \prime} \subset \operatorname{rad} A^{\prime \prime}$, the conclusion follows by Lemma 3.1.

By Corollary 3.2 we can make the set of all $N$-extensions of $(A, \mathfrak{m})$ into a semiordered set: if $\left(A^{\prime}, \mathfrak{m}^{\prime}\right)\left(A^{\prime \prime}, \mathfrak{m}^{\prime \prime}\right)$ are two $N$-extensions of $(A, \mathfrak{m})$ we say $\left(A^{\prime}, \mathfrak{m}^{\prime}\right)$ $\leqq\left(A^{\prime \prime}, \mathfrak{m}^{\prime \prime}\right)$ if and only if there is a morphism $\psi:\left(A^{\prime}, \mathfrak{m}^{\prime}\right) \rightarrow\left(A^{\prime \prime}, \mathfrak{m}^{\prime \prime}\right)$ such that diagram (1) above is commutative. This is a good definition by Corollary 3.2.

Now we prove:

Proposition 3.3. Let $(A, \mathfrak{m})$ be a pair. Then the $N$-extensions of $(A, \mathfrak{m})$ form a direct set (with the order relation and the morphisms defined above).

Proof. It is clear that all the $N$-extensions of $(A, \mathfrak{m})$ form a set, and that the order relation-defined above is transitive and agrees with the morphisms. Therefore it remains to show that if $\left(A_{i}, \mathfrak{m}_{i}\right), i=1,2$, are two $N$-extensions of $(A, \mathfrak{m})$, there is an $N$-extension $(B, \mathfrak{n})$ of $(A, \mathfrak{m})$ such that $\left(A_{\mathfrak{i}}, \mathfrak{m}_{\mathfrak{i}}\right) \leqq(B, \mathfrak{n}), i=1,2$.

Suppose first $\left(A_{i}, \mathfrak{m}_{i}\right)$ are simple $N$-extensions, and let $F_{1}(X)$ be an $N$-polynomial defining $\left(A_{1}, \mathfrak{m}_{1}\right)$ (Definition 2.2). Let $G_{1}(X)$ be the image of $F_{1}(X)$ in $A_{2}[X]$. Then $G_{1}(X)$ is an $N$-polynomial over $\left(A_{2}, \mathfrak{m}_{2}\right)$ (Proposition 6.2). Let $(B, \mathfrak{n})$ be the simple $N$-extension of $\left(A_{2}, \mathfrak{m}_{2}\right)$ constructed with $G_{1}(X)$ (Definition 2.2). Then $(B, \mathfrak{n})$ is an $N$-extension of $(A, \mathfrak{m})$, and it is not difficult to see that $\left(A_{i}, \mathfrak{m}_{\mathfrak{i}}\right) \leqq$ $(B, \mathfrak{n}), i=1,2$. Thus the conclusion follows in this case.

The general case follows by repeating a finite number of times the above argument. 
By Proposition 3.3 the direct limit of the set of $N$-extensions of $(A, \mathfrak{m})$ exists. We will prove that it is the $N$-closure of $(A, \mathfrak{m})$ (Definition 1.7).

LEMMA 3.4. Let $\left(A^{\prime}, \mathfrak{m}^{\prime}\right)$ be an $N$-extension of $(A, \mathfrak{m})$, and let $\phi:(A, \mathfrak{m}) \rightarrow\left(A^{\prime}, \mathfrak{m}^{\prime}\right)$ be the canonical morphism. Let $(B, \mathfrak{n})$ be an $N$-pair (Definition 1.3), and let $\psi:(A, \mathfrak{m})$ $\rightarrow(B, \mathfrak{n})$ be a morphism. Then there is a unique morphism $\psi^{\prime}:\left(A^{\prime}, \mathfrak{m}^{\prime}\right) \rightarrow(B, \mathfrak{n})$ such that $\psi \circ \phi=\psi$.

Proof. We may assume $\left(A^{\prime}, \mathfrak{m}^{\prime}\right)$ is a simple $N$-extension. Let $F(X)$ be an $N$ polynomial over $(A, \mathfrak{m})$ used to construct $\left(A^{\prime}, \mathfrak{m}^{\prime}\right)$ (Definition 2.2), and let $G(X)$ $=\psi(F(X))$. Then $G(X)$ is an $N$-polynomial over $(B, \mathfrak{n})$, and therefore it has a root $y \in \mathfrak{n}$ (Definition 1.3). Then there is a ring homomorphism $\rho: A[x]=A[X] /(F(X))$ $\rightarrow B$ such that $\rho(x)=y$ and $\left.\rho\right|_{A}=\psi$. Since $\psi(\mathfrak{m}) \subset \mathfrak{n}$ and $y \in \mathfrak{n}$ we have $\rho((\mathfrak{m}, x) A[x])$ $\subset \mathfrak{n}$. But $\mathfrak{n} \subset \operatorname{rad} B$, and hence every element of $1+\mathfrak{n}$ is invertible. Therefore $\rho$ extends to $\psi^{\prime}: S^{-1} A[x] \rightarrow B$, where $S=1+(\mathfrak{m}, x) A[x]$. But $S^{-1} A[x]$ is just $A^{\prime}$ by definition, and since $x \in \mathfrak{m} A^{\prime}=\mathfrak{m}^{\prime}$ (Lemma 2.3) it is easy to see that $\psi^{\prime}$ is actually a morphism $\psi^{\prime}:\left(A^{\prime}, \mathfrak{m}^{\prime}\right) \rightarrow(B, \mathfrak{n})$. The equality $\psi^{\prime} \circ \phi=\psi$ is obvious, and unicity follows by Lemma 3.1. This completes the proof.

TheOREM 3.5. Let $(A, \mathfrak{m})$ be a pair, and let $\left({ }^{n} A,{ }^{n} \mathfrak{m}\right)$ be the direct limit of the set of all $N$-extensions of $(A, \mathfrak{m})$ (Proposition 3.3). Then $\left({ }^{n} A,{ }^{n} \mathfrak{m}\right)$, with the canonical morphism $(A, \mathfrak{m}) \rightarrow\left({ }^{n} A,{ }^{n} \mathfrak{m}\right)$, is the $N$-closure of $(A, \mathfrak{m})$ (Definition 1.7).

Proof. It is clear by construction that $\left({ }^{n} A,{ }^{n} \mathfrak{m}\right)$ is an $N$-pair. It remains to show that if $\psi:(A, \mathfrak{m}) \rightarrow(B, \mathfrak{n})$ is a morphism, and $(B, \mathfrak{n})$ is an $N$-pair, there is a unique morphism ${ }^{n} \psi:\left({ }^{n} A,{ }^{n} \mathfrak{m}\right) \rightarrow(B, \mathfrak{n})$ such that the following diagram is commutative:

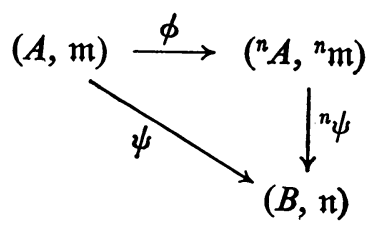

where $\phi$ is the canonical morphism.

Let $\left(A_{i}, \mathfrak{m}_{i}\right)_{i \in I}$ be the direct set of all $N$-extensions of $(A, \mathfrak{m})$, so that, by assumption, $\left({ }^{n} A,{ }^{n} \mathfrak{m}\right)=\operatorname{inj} \lim \left(A_{i}, \mathfrak{m}_{i}\right)$. By Lemma 3.4 we have, for every $i$, a unique morphism $\psi_{i}:\left(A_{i}, \mathfrak{m}_{i}\right) \rightarrow(B, \mathfrak{n})$ such that the following diagram is commutative:

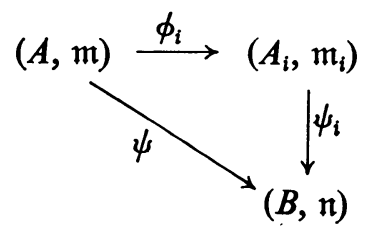


where $\phi_{i}$ are the canonical morphisms. It is then clear that ${ }^{n} \psi=\operatorname{inj} \lim \psi_{i}$ is the unique morphism $\left({ }^{n} A,{ }^{n} \mathfrak{m}\right) \rightarrow(B, \mathfrak{n})$ such that diagram (2) is commutative. This completes the proof.

By Theorem 3.5 we have immediately:

COROllary 3.6. Let $\left(A_{i}, \mathfrak{m}_{i}\right)_{i \in I}$ be a direct set of pairs, and let $(A, \mathfrak{m})=$ inj $\lim \left(A_{\mathfrak{i}}, \mathfrak{m}_{\mathfrak{i}}\right)$. Then $\left({ }^{n} A_{\mathfrak{i}},{ }^{n} \mathfrak{m}_{\mathfrak{i}}\right)_{\mathfrak{i} \in I}$ is a direct set, and $\left({ }^{n} A,{ }^{n} \mathfrak{m}\right)=\operatorname{inj} \lim \left({ }^{n} A_{i},{ }^{n} \mathfrak{m}_{\mathfrak{i}}\right)$.

COROLlaRY 3.7. The canonical morphism of a pair in its $N$-closure is strict (Definition 2.1).

Proof. Since a direct limit of strict morphisms is clearly strict, the conclusion follows by Theorem 3.5 and Proposition 2.6.

Corollary 3.8. Let $\left({ }^{n} A,{ }^{n} \mathfrak{m}\right)$ be the $N$-closure of $(A, \mathfrak{m})$. Then ${ }^{n} A$ is flat over $A$.

Proof. Since a direct limit of flat $A$-modules is a flat $A$-module, the conclusion follows immediately by

Lemma 3.9. Let $(B, \mathfrak{n})$ be an $N$-extension of $(A, \mathfrak{m})$. Then $B$, is flat over $A$.

Proof. We may clearly assume that $(B, \mathfrak{n})$ is a simple $N$-extension. $[2$, p. 35, Corollary 3]. Then we have $B=S^{-1} A[x]$ where $A[x]=A[X] /(F(X)), F(X)$ an $N$-polynomial over $(A, \mathfrak{m})$ (Definition 2.2). Since $F(X)$ is monic it is easy to show that $A[x]$ is a free $A$-module. Since $B$ is flat over $A[x][2, \mathrm{p} .88$, Theorem 1$]$ the conclusion follows.

4. The aim of this and the following section is to show that the $N$-closure of a pair, constructed in $\$ 3$, coincides with the $H$-closure (Theorem 5.10), and that $H$-pairs and $N$-pairs are the same thing (Theorem 5.11). In order to do that, we have to study some general properties of the $N$-pairs, and the structure of the $N$-closure of a noetherian ring.

In this section we deal with the first argument, and the main result we get is Proposition 4.10, which is a first generalization of

Proposition 4.1 [6, Theorem 6.1]. Let $(A, \mathfrak{m})$ be an $N$-pair, and suppose

(i) $A$ is normal,

(ii) $\mathfrak{m}=\sqrt{ } \mathfrak{m}$,

(iii) $\operatorname{spec}(A / \mathfrak{m})$ is connected.

Then $(A, \mathfrak{m})$ is an $H$-pair.

We will show in this section that Proposition 4.1 is true for a direct sum of finitely many normal rings, and any ideal.

We begin with some remarks on decompositions of an $N$-pair; the aim of this preliminary discussion is Proposition 4.7.

LEMMA 4.2. Let $(A, \mathfrak{m})$ be an $N$-pair. Then every idempotent of $A / \mathfrak{m}$ is the image of a unique idempotent of $A$. 
Proof. It follows by the definition and a standard argument (see e.g. [7, Proof of Proposition 1.7]).

Corollary 4.3. Let $(A, \mathfrak{m})$ be an $N$-pair. Then for every decomposition $A / \mathfrak{m}$ $=B_{1} \oplus \cdots \oplus B_{n}$ (direct sum of rings), there is a unique decomposition $A=A_{1} \oplus \cdots$ $\oplus A_{n}$ such that $A_{\mathfrak{i}} / \mathfrak{m} A_{i}=B_{i}$ for every $i=1, \ldots, n$.

Proof. It follows by Lemma 4.2 and a well-known technique (see e.g. [3, p. 96, Proposition 8]).

By Lemma 4.2 and $[2$, p. 132, Corollary 2] we get

COROllary 4.4. Let $(A, \mathfrak{m})$ be an $N$-pair. Then spec $(A)$ is connected if and only if $\operatorname{spec}(A / \mathfrak{m})$ is connected.

Corollary 4.5. Let $(A, \mathfrak{m})$ be an $N$-pair, and suppose $A$ is normal and $\mathfrak{m}=\sqrt{ } \mathfrak{m}$. Then $(A, \mathfrak{m})$ is an $H$-pair.

Proof. Since $A$ is a domain, $\operatorname{spec}(A / \mathfrak{m})$ is connected (Corollary 4.4), and the conclusion follows by Proposition 4.1.

Now we want to generalize Corollary 4.5 to a direct sum of normal domains, and to drop the hypothesis $\mathfrak{m}=\sqrt{ } \mathfrak{m}$. To solve the first problem we need:

Lemma 4.6. Let $(A, \mathfrak{m})$ be a pair, and suppose $A=A_{1} \oplus A_{2}$ (direct sum of rings). Then $(A, \mathfrak{m})$ is an $N$-pair if and only if $\left(A_{i}, \mathfrak{m} A_{\mathfrak{i}}\right)$ is an $N$-pair $(i=1,2)$.

Proof. Suppose $(A, \mathfrak{m})$ is an $N$-pair. Then $\mathfrak{m} \subset \operatorname{rad} A$ (Definition 1.3) and hence $\mathfrak{m} A_{i} \subset \operatorname{rad} A_{i}(i=1,2)$. Let now $F(X)$ be an $N$-polynomial over $\left(A_{1}, \mathfrak{m} A_{1}\right)$. We want to show that $F$ has a root in $\mathfrak{m} A_{1}$. Let $1=e_{1}+e_{2}, e_{i} \in A_{i}(i=1,2)$. Then it is easy to see that there is an $N$-polynomial $G(X)$ over $(A, \mathfrak{m})$ such that $F(X)=e_{1} G(X)$. By assumption there is an $m \in \mathfrak{m}$ such that $G(m)=0$, and it is easy to see that we have $F\left(e_{1} m\right)=e_{1} G\left(e_{1} m\right)=e_{1} G(m)=0$. Then $\left(A_{1}, \mathfrak{m} A_{1}\right)$ is an $N$-pair, and similarly for $\left(A_{2}, \mathfrak{m} A_{2}\right)$.

Conversely suppose $\left(A_{i}, \mathfrak{m} A_{i}\right)$ is an $N$-pair $(i=1,2)$. If $F(X)$ is an $N$-polynomial over $(A, \mathfrak{m})$, then $e_{i} F(X)$ is an $N$-polynomial over $\left(A_{i}, \mathfrak{m} A_{i}\right)$, whence there is $m_{i} \in \mathfrak{m} A_{i}$ such that $e_{i} F\left(m_{i}\right)=0(i=1,2)$. Since $e_{1}, e_{2}$ are orthogonal idempotents, it is easy to see that $m=m_{1}+m_{2}$ is a solution of $F(X)$ contained in $m$. It is also clear that $\mathfrak{m} \subset \operatorname{rad} A$, whence $(A, \mathfrak{m})$ is an $N$-pair.

By Corollary 4.3 and Lemma 4.6 we have immediately

Proposition 4.7. Let $(A, \mathfrak{m})$ be an $N$-pair, and suppose $A / \mathfrak{m}=B_{1} \oplus \cdots \oplus B_{n}$, (direct sum of rings). Then there are rings $A_{1}, \ldots, A_{n}$ such that

(i) $A=A_{1} \oplus \cdots \oplus A_{n}$

(ii) $A_{i} / \mathfrak{m} A_{i}=B_{i}(i=1, \ldots, n)$

(iii) $\left(A_{i}, \mathfrak{m} A_{i}\right)$ is an $N$-pair $(i=1, \ldots, n)$.

Now we deal with hypothesis (ii) of Proposition 4.1. This hypothesis can be dropped by Corollary 4.9 below. 
Proposition 4.8. Let $(A, \mathfrak{m})$ be a pair, and let $\mathfrak{n}$ be an ideal of $A$ contained in $\mathfrak{m}$. Then the following conditions are equivalent:

(i) $(A, \mathfrak{m})$ is an $N$-pair.

(ii) $(A, \mathfrak{n})$ and $(A / \mathfrak{n}, \mathfrak{m} / \mathfrak{n})$ are $N$-pairs.

Proof. Suppose $(A, \mathfrak{m})$ is an $N$-pair; then it is clear that $(A / \mathfrak{n}, \mathfrak{m} / \mathfrak{n})$ is an $N$-pair, since every $N$-polynomial over $(A / \mathfrak{n}, \mathfrak{m} / \mathfrak{n})$ can be lifted to an $N$-polynomial over $(A, \mathfrak{m})$, and $\mathfrak{m} / \mathfrak{n} \subset \operatorname{rad} A / \mathfrak{n}$.

Moreover let $a_{0}+a_{1} X+\cdots+X_{n}=F(X)$ be an $N$-polynomial over $(A, \mathfrak{n})$. Then $F(X)$ is also an $N$-polynomial over $(A, \mathfrak{m})$, whence there is $x \in \mathfrak{m}$ such that $F(x)=0$. Then $x\left(a_{1}+a_{2} x+\cdots+x^{n-1}\right)=-a_{0} \in \mathfrak{n}$, and since $a_{1}$ is a unit, and $x \in \mathfrak{m} \subset \operatorname{rad} A$, it follows that $a_{1}+a_{2} x+\cdots+x^{n-1}$ is a unit, and $x \in \mathfrak{n}$. Thus $(A, \mathfrak{n})$ is an $N$-pair.

Conversely suppose (ii) holds. Then one sees easily that $\mathfrak{m} \subset \operatorname{rad} A$. Let now $F(X)=a_{0}+a_{1} X+\cdots+X^{n}$ be an $N$-polynomial over $(A, \mathfrak{m})$, and let $\bar{F}(X)$ be the reduction of $F$ modulo $\mathfrak{n}$. Then $\bar{F}(X)$ is an $N$-polynomial over $(A / \mathfrak{n}, \mathfrak{m} / \mathfrak{n})$, whence there is an $x \in \mathfrak{m}$ such that $\bar{F}(\bar{x})=0$.

Let $G(Y)=F(x+Y)$. Then we have, for suitable $b_{2}, \ldots, b_{n-1}, c \in A$

$$
G(Y)=F(x)+\left(a_{1}+c x\right) Y+b_{2} Y^{2}+\cdots+Y^{n} .
$$

Since $F(x) \in \mathfrak{n}$ and $a_{1}+c x$ is invertible, $G(Y)$ is an $N$-polynomial over $(A, \mathfrak{n})$, whence there is a $y \in \mathfrak{n}$ such that $G(y)=0$. Therefore we get $F(x+y)=0$, and since $x+y \in \mathfrak{m}$, the proof is complete.

COROllary 4.9. Let $A$ be a ring and $\mathfrak{m}$ an ideal of $A$. Then $(A, \mathfrak{m})$ is an $N$-pair if and only if $(A, \sqrt{ } \mathfrak{m})$ is an $N$-pair.

Proof. Since $\sqrt{ } \mathfrak{m} / \mathfrak{m}$ is a nil ideal of $A / \mathfrak{m},(A / \mathfrak{m}, \sqrt{ } \mathfrak{m} / \mathfrak{m})$ is an $H$-pair [3, p. 84, Theorem 1] and hence an $N$-pair (Lemma 1.4). The conclusion follows by Proposition 4.8.

Now we can give a first generalization of Proposition 4.1, which was announced in [5] (Theorem 2.6), without proof.

Proposition 4.10: Let $(A, \mathfrak{m})$ be a pair and suppose $A=A_{1} \oplus \cdots \oplus A_{n}$, where $A_{1}, \ldots, A_{n}$ are normal domains. Then $(A, \mathfrak{m})$ is an $H$-pair if and only if it is an $N$-pair.

Proof. Assume $(A, \mathfrak{m})$ is an $N$-pair. Then $\left(A_{\mathfrak{i}}, \mathfrak{m} A_{\mathfrak{i}}\right)$ is an $N$-pair (Lemma 4.6), and so is $\left(A_{i}, \sqrt{ }\left(\mathfrak{m} A_{i}\right)\right)$ (Corollary 4.9). Since. $A_{i}$ is normal, $\left(A_{i}, \sqrt{ }\left(\mathfrak{m} A_{i}\right)\right)$ is an $H$-pair (Corollary 4.5); hence $\left(A_{i}, \mathfrak{m} A_{i}\right)$ is an $H$-pair [7, Corollary 4.2]. This holds for every $i=1, \ldots, n$, and a straightforward computation shows that $(A, \mathfrak{m})$ is an $H$-pair.

The converse follows by Lemma 1.4 .

CoRollary 4.11. Let $(A, \mathfrak{m})$ be an $N$-pair. If $A$ is a regular ring, $(A, \mathfrak{m})$ is an H-pair. 
Proof. By assumption we have $A=A_{1} \oplus \cdots \oplus A_{n}$, where $A_{i}$ is a regular domain for every $i=1, \ldots, n$. Since regular domains are normal the conclusion follows by Proposition 4.10.

5. In this section we show that the $N$-closure of a pair coincides with its $H$ closure (Theorem 5.10). To obtain this result we shall use Corollary 4.11, and in order to do that we have to show first that the $N$-closure of a regular ring is regular (Proposition 5.5), and to study the $N$-closure of a quotient of a regular ring (Lemma 5.8, Corollary 5.9). The results we are seeking will follow easily by reducing the general problem to the above situation by using direct limits.

To show that the $N$-closure of a regular ring is regular, we compare $N$-closure and completions. For this we need

Lemma 5.0. Let $\phi:(A, \mathfrak{m}) \rightarrow(B, \mathfrak{n})$ be a strict morphism (Definition 2.1), and suppose $\mathfrak{n} \subset \operatorname{rad} B$. Then the following conditions are equivalent

(i) $B$ is faithfully flat over $A$.

(ii) $B$ is flat over $A$, and $\mathfrak{m} \subset \operatorname{rad} A$.

Proof. Suppose $B$ is faithfully flat over $A$. Then for each maximal ideal $\mathfrak{p}$ of $A$ there is a maximal ideal $\mathfrak{q}$ of $B$ such that $\mathfrak{q} \cap A=\mathfrak{p}$ [2, p. 51, Proposition 9]. Since $\mathfrak{n} \subset \mathfrak{q}$ and $\phi$ is strict, we see easily that $\mathfrak{m} \subset \mathfrak{p}$. Thus $\mathfrak{m} \subset \operatorname{rad} A$, and (i) $\Rightarrow$ (ii). To prove the converse it is sufficient to show that for each maximal ideal $\mathfrak{p}$ of $A$, $\mathfrak{p} B \neq B[2$, p. 44, Proposition 1]. But this follows easily since $\mathfrak{m} \subset \operatorname{rad} A$ and $\phi$ is strict.

LEMMA 5.1. Let $\phi:(A, \mathfrak{m}) \rightarrow(B, \mathfrak{n})$ be a strict flat morphism (Definition 2.1). Then

(i) The induced homomorphisms $\phi_{k}: A / \mathfrak{m}^{k} \rightarrow B / \mathfrak{n}^{k}$ are bijective $(k=1,2, \ldots)$.

(ii) The canonical morphism $\hat{\phi}:(\hat{A}, \hat{\mathfrak{m}}) \rightarrow(\hat{B}, \hat{\mathfrak{n}})$ is bijective.

Proof. Since $\mathfrak{n}=\mathfrak{m} B$ we have for every $k$,

$$
\mathfrak{n} / \mathfrak{n}^{k}=\mathfrak{m}\left(B / \mathfrak{n}^{k}\right)
$$

Let $\bar{A}=A / \mathfrak{m}^{k}, B=B / \mathfrak{n}^{k}$. Then by (1) we have

$$
\bar{A} / \mathfrak{m} \bar{A} \cong \bar{A} / \mathfrak{m} \cong \bar{B} / \mathfrak{n} \cong \bar{B} / \mathfrak{m} \bar{B} .
$$

Moreover $\mathfrak{m} \bar{A}$ is a nilpotent ideal, and then $\phi_{k}$ is onto (as follows easily by [2, p. 105, Proposition 4]). We clearly assume $\mathfrak{m} \subset \operatorname{rad} A$, by localizing at the multiplicative set $1+\mathfrak{m}$. Thus $B$ is faithfully flat over $A$ (Lemma 5.0), whence $\mathfrak{n}^{k} \cap A=\mathfrak{n}^{k} B \cap A=\mathfrak{m}^{k}$ [2, p. 51, Proposition 9], and $\phi_{k}$ is injective. This proves (i).

By (i) we have $\hat{A}=\operatorname{proj} \lim A / \mathfrak{m}^{k}=\operatorname{proj} \lim B / \mathfrak{n}^{k}=\hat{B}$, whence $\hat{\phi}: \hat{A} \rightarrow \hat{B}$ is an isomorphism. Moreover $\hat{A} / \hat{\mathfrak{m}}=A / \mathfrak{m}=B / \mathfrak{n}=\hat{B} / \hat{\mathfrak{n}}$ [3, p. 50, Proposition 15], whence $\hat{\phi}$ is a morphism of pairs. This proves (ii) and concludes the proof.

Proposition 5.2. Let $\left({ }^{n} A,{ }^{n} \mathfrak{m}\right)$ be the $N$-closure of $(A, \mathfrak{m})$, and let $\phi:(A, \mathfrak{m})$ $\rightarrow\left({ }^{n} A,{ }^{n} \mathfrak{m}\right)$ be the canonical morphism. Then

(i) $\phi$ is strict and flat.

(ii) $\hat{\phi}:(A, \mathfrak{m})^{\wedge} \rightarrow\left({ }^{n} A,{ }^{n} \mathfrak{m}\right)^{\wedge}$ is an isomorphism. 
Proof. By Corollaries 3.7 and 3.8 we have (i), and (ii) follows then by (i) and Lemma 5.1.

Now we want to show that if $A$ is noetherian so is ${ }^{n} A$. To do that we will show that $\hat{A}$ is faithfully flat over ${ }^{n} A$ (Proposition 5.5).

We need the following:

LEMmA 5.3. Let $(B, \mathfrak{n})$ be an $N$-extension of $(A, \mathfrak{m})$, and suppose $A$ is noetherian. Then $B$ is canonically embedded in $\hat{A}$ (the $\mathfrak{m}$-completion of $A$ ) and $\hat{A}$ is faithfully flat over $B$.

Proof. Since the canonical morphism $\phi:(A, \mathfrak{m}) \rightarrow(B, \mathfrak{n})$ is strict (Proposition 2.6) and flat (Lemma 3.9), by Lemma 5.1 we have that $\hat{\phi}:(\hat{A}, \hat{\mathfrak{m}}) \rightarrow(\hat{B}, \hat{\mathfrak{n}})$ is an isomorphism. Moreover since $A$ is noetherian, it follows immediately by Definition 2.2 that $B$ is noetherian. Moreover $\mathfrak{n} \subset \operatorname{rad} B$ by construction, and then $B$ in a Zariski ring for the $n$-topology. Therefore $B$ is faithfully flat over $B[3$, p. 72 , Proposition 9], and since $\hat{A}=\hat{B}$ the conclusion follows.

LEMMA 5.4. Let $B$ be $a$ ring and $\left\{A_{i}\right\}_{i \in I}$ be a direct set of subrings of $B$, and let $A=\operatorname{inj} \lim A_{i}$. If $B$ is (faithfully) flat over $A_{i}$ for every $i \in I$, then $B$ is (faithfully) flat over $A$.

Proof. It is known that flatness and faithful flatness can be characterized in terms of linear relations, each involving only a finite number of coefficients of $A$ [2, p. 44, Corollary 2, and p. 54, Proposition 13]. Since for every finite set $E$ of elements of $A$ there is an $i \in I$ such that $E \subset A_{i}$, the conclusion follows easily by the above characterization.

Proposition 5.5. Let $(B, \mathfrak{n})$ be the $N$-closure of $(A, \mathfrak{m})$, and suppose $A$ is noetherian. Then

(i) $B$ is a subring of $\hat{A}$, and $\hat{A}$ is faithfully flat over $B$.

(ii) $B$ is noetherian.

(iii) If $A$ is regular so is $B$.

Proof. By Theorem 3.5 we have $(B, \mathfrak{n})=\operatorname{inj} \lim \left(A_{i}, \mathfrak{m}_{\mathfrak{i}}\right)$ where $\left(A_{i}, \mathfrak{m}_{\mathfrak{i}}\right)$ is the direct set of all $N$-extensions of $(A, \mathfrak{m})$. Since $A$ is faithfully flat over $A_{i}$ for every $i$ (Lemma 5.3), it follows that $\hat{A}$ is faithfully flat over $B$ (Lemma 5.4). This proves (i); and since $\hat{A}$ is noetherian, $B$ is noetherian [2, p. 50, Corollary], and this proves (ii).

By Proposition 5.2 we have $(\hat{A}, \hat{\mathfrak{m}})=(\hat{B}, \hat{\mathfrak{n}})$, and since $A$ and $B$ are noetherian and $\mathfrak{n} \subset \operatorname{rad} B$, (iii) follows easily by:

LEMMA 5.6. Let $B$ be a noetherian ring, $\mathfrak{n}$ an ideal of $B$ and $\hat{B}$ the $\mathfrak{n}$-adic completion of $B$. Then

(i) If $B$ is regular, $\hat{B}$ is regular.

(ii) If $B$ is regular and $\mathfrak{n} \subset \operatorname{rad} B, B$ is regular.

Proof. See e.g. [8, p. 80, Proof of Lemma 17.3.8.1].

By Proposition 5.5 and Corollary 4.11 we have 
Corollary 5.7. Let $A$ be a regular ring and $\mathfrak{m}$ an ideal of $A$. Then the $N$-closure of $(A, \mathfrak{m})$ is an H-pair.

Now we generalize Corollary 5.7 to quotients of regular rings. In order to do this we need:

LEMMA 5.8. Let $A$ be a noetherian ring, $\mathfrak{a} \subset \mathfrak{m}$ two ideals of $A, B=A / \mathfrak{a}$ and $\mathfrak{n}=\mathfrak{m} / \mathfrak{a}$. Then there is a canonical isomorphism

$$
\rho:\left({ }^{n} A /{ }^{n} \mathfrak{a},{ }^{n} \mathfrak{m} /{ }^{n} \mathfrak{a}\right) \rightarrow\left({ }^{n} B,{ }^{n} \mathfrak{n}\right) .
$$

(We denote by ${ }^{n} \mathfrak{a}$ the extension of $\mathfrak{a}$ to ${ }^{n} A$.)

Proof. Let $\pi:(A, \mathfrak{m}) \rightarrow(B, \mathfrak{n})$ be the canonical morphism. By the universal property of $N$-closure, $\pi$ induces a morphism

$$
{ }^{n} \pi:\left({ }^{n} A,{ }^{n} \mathfrak{m}\right) \rightarrow\left({ }^{n} B,{ }^{n} \mathfrak{n}\right) .
$$

Since $\mathfrak{a} \subset \mathfrak{m}$, every $N$-polynomial over $(B, \mathfrak{n})$ is the image of an $N$-polynomial over $(A, \mathfrak{m})$, and then it follows easily by Theorem 3.5 that ${ }^{n} \pi$ is onto.

To complete the proof we have to show that $\operatorname{Ker}\left({ }^{n} \pi\right)={ }^{n} \mathfrak{a}$. Since $A$ and $B$ are noetherian, ${ }^{n} A$ and ${ }^{n} B$ are noetherian (Proposition 5.5(i)). Moreover by Proposition 5.2 we have $\hat{A}=\left({ }^{n} A\right)^{\wedge}$ and $B=\left({ }^{n} B\right)^{\wedge}$. Since $\hat{B}=B \otimes_{A} \hat{A}=\hat{A} / \mathfrak{a} \hat{A}$ we get a commutative diagram

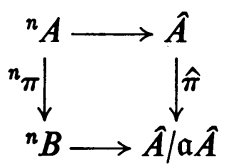

By Proposition 5.5(ii) $\hat{A}$ is faithfully flat over ${ }^{n} A$, whence [2, p. 51, Proposition 9]

$$
\begin{aligned}
\operatorname{ker}\left({ }^{n} \pi\right) & =(\operatorname{ker} \hat{\pi}) \cap\left({ }^{n} A\right)=\mathfrak{a} \hat{A} \cap\left({ }^{n} A\right) \\
& =\left({ }^{n} \mathfrak{a}\right) \hat{A} \cap\left({ }^{n} A\right)={ }^{n} \mathfrak{a},
\end{aligned}
$$

and the proof is complete.

Corollary 5.9. Let $A$ be a regular ring, $\mathfrak{a} \subset \mathfrak{m}$ two ideals of $A, B=A / \mathfrak{a}$ and $\mathfrak{n}=\mathfrak{m} / \mathfrak{a}$. Then the $N$-closure of $(B, \mathfrak{n})$ is an H-pair.

Proof. By Corollary 5.7 the $N$-closure $\left({ }^{n} A,{ }^{n} \mathfrak{m}\right)$ of $(A, \mathfrak{m})$ is an $H$-pair. Therefore $\left({ }^{n} A /{ }^{n} \mathfrak{a},{ }^{n} \mathfrak{m} /{ }^{n} \mathfrak{a}\right)$ is an $H$-pair [7, Theorem 4.6], and the conclusion follows by Lemma 5.8.

Now we can prove

THEOREM 5.10. Let $(A, \mathfrak{m})$ be a pair. Then the $N$-closure of $(A, \mathfrak{m})$ is an H-pair, and is, in fact, the henselization of $(A, \mathfrak{m})$.

Proof. If the $N$-closure of $(A, \mathfrak{m})$ is an $H$-pair, the theorem follows easily by Lemma 1.4 and the definitions (Definition 1.7). 
To conclude our proof we reduce the problem to Corollary 5.9 as follows. Let $\left\{A_{i}\right\}$ be the direct set of all finitely generated sub- $Z$-algebras of $A$, and let $\mathfrak{m}_{i}$ $=\mathfrak{m} \cap A_{i}$. Then $(A, \mathfrak{m})=\operatorname{inj} \lim \left(A_{i}, \mathfrak{m}_{i}\right)$ whence $\left({ }^{n} A,{ }^{n} \mathfrak{m}\right)=\operatorname{inj} \lim \left({ }^{n} A_{i},{ }^{n} \mathfrak{m}_{i}\right)$ (Corollary 3.6). By Corollary $5.9\left({ }^{n} A_{i},{ }^{n} \mathfrak{m}_{i}\right)$ is an $H$-pair, whence the conclusion, since a direct limit of $H$-pairs is an $H$-pair [10, Proposition 1].

By Theorem 5.10 and Lemma 1.4 we have immediately a complete generalization of Proposition 4.1:

THEOREM 5.11. A pair is an H-pair if and only if it is an-pair.

REMARK 5.12. Theorem 5.10 states that the henselization exists. This fact was proved by J. P. Lafon by a different technique [10, Theorem 1]. Our theorem, however, gives an explicit construction of the hensilization, which will be very useful to investigate many properties of henselization, as we will do in the rest of this paper.

6. In this section we give a first set of applications of Theorem 5.10. After summarizing the main results obtained so far in the previous sections (Theorem 6.1), we state a theorem of flatness (Theorem 6.5). We show then that the henselization of a noetherian ring is noetherian (Theorem 6.8), and that it coincides with its "relative henselization" (Theorem 6.11).

THEOREM 6.1. Let $(A, \mathfrak{m})$ be a pair. Then

(i) The henselization $\left({ }^{h} A,{ }^{h} \mathfrak{m}\right)$ of $(A, \mathfrak{m})$ exists, and is unique up to isomorphism.

(ii) $\left({ }^{h} A,{ }^{n} \mathfrak{m}\right)=\operatorname{inj} \lim \left(A_{\mathfrak{i}}, \mathfrak{m}_{i}\right)$ where $\left(A_{\mathfrak{i}}, \mathfrak{m}_{\mathfrak{i}}\right), i \in l$, is the direct set of the $N$-extensions of $(A, \mathfrak{m})$ (Definition 2.2 and Proposition 3.3). In other words $\left({ }^{n} A,{ }^{n} \mathfrak{m}\right)$ coincides with the $N$-closure of $(A, \mathfrak{m})$ (Definition 1.7).

(iii) The canonical morphism $\phi:(A, \mathfrak{m}) \rightarrow\left({ }^{h} A,{ }^{h} \mathfrak{m}\right)$ is strict and flat (Definition 2.1).

(iv) $\phi$ extends uniquely to an isomorphism $\hat{\phi}:(\hat{A}, \hat{\mathfrak{m}}) \rightarrow\left({ }^{h} A,{ }^{h} \mathfrak{m}\right)^{\wedge}$.

Proof. By Theorem 5.10 the henselization of $(A, \mathfrak{m})$ exists and is the $N$-closure of $(A, \mathfrak{m})$, i.e. the direct limit of the $N$-extensions of $(A, \mathfrak{m})$. This proves (i) and (ii), while (iii) and (iv) follow by Theorem 5.10 and Proposition 5.2.

REMARK 6.2. Theorem 6.1 is due to Nagata when $A$ is a local ring and $\mathfrak{m}$ is its maximal ideal [13, Chapter VII, 11 and 12]. As for general pairs, the existence of henselization and the strictness of the canonical morphism $\phi$ were proved, in a different way, by Lafon (see [10]), while the flatness of $\phi$ was announced in [5].

Now we give some easy consequences of Theorem 6.1. Recall first that by Corollary 3.6 we have

COROLlary 6.3. Henselization commutes with direct limits.

The next corollary sharpens a result by Lafon [10, Corollary 2 to Theorem 1].

COROLlaRy 6.4. Let $(B, \mathfrak{n})$ be the henselization of $(A, \mathfrak{m})$, and let $\mathfrak{a} \subset \mathfrak{m}$ be an ideal of $A$. Then the henselization of $(A / \mathfrak{a}, \mathfrak{m} / \mathfrak{a})$ is $(B / \mathfrak{a} B, \mathfrak{n} / \mathfrak{a} B)$. 
Proof. If $A$ is noetherian the conclusion follows by Theorem 6.1 and Lemma 5.8. To prove the general case, consider the direct set $\left\{A_{i}\right\}_{i \in I}$ of all finitely generated sub-Z-algebras of $A$. For every $i \in I$ let $\mathfrak{m}_{i}=\mathfrak{m} \cap A_{i}, \mathfrak{a}_{i}=\mathfrak{a} \cap A_{i}$. Then

$$
(A, \mathfrak{m})=\operatorname{inj} \lim \left(A_{\mathfrak{i}}, \mathfrak{m}_{\mathfrak{i}}\right) \text { and }(A / \mathfrak{a}, \mathfrak{m} / \mathfrak{a})=\operatorname{inj} \lim \left(A_{\mathfrak{i}} / \mathfrak{a}_{\mathfrak{i}}, \mathfrak{m}_{\mathfrak{i}} / \mathfrak{a}_{\mathfrak{i}}\right) \text {. }
$$

Let $\left(B_{i}, \mathfrak{n}_{i}\right)$ be the henselization of $\left(A_{i}, \mathfrak{m}_{i}\right)$. Since the $A_{i}$ 's are noetherian, by the above remark $\left(B_{i} / \mathfrak{a}_{i} B_{i}, \mathfrak{n}_{\mathfrak{i}} / \mathfrak{a}_{\mathfrak{i}} B_{i}\right)$ is the henselization of $\left(A_{\mathfrak{i}} / \mathfrak{a}_{i}, \mathfrak{m}_{i} / \mathfrak{a}_{\mathfrak{i}}\right)$. Since henselization commutes with direct limits (Corollary 6.3) the conclusion follows easily.

Bỳ Theorem 6.1 (iii) and Lemma 5.0 we have

THEOREM 6.5. Let $\left({ }^{n} A,{ }^{n} \mathfrak{m}\right)$ be the henselization of $(A, \mathfrak{m})$. Then

(i) ${ }^{h} A$ is flat over $A$.

(ii) ${ }^{h} A$ is faithfully flat over $A$ if and only if $m \subset \operatorname{rad} A$.

Now we apply Theorem 6.1 to noetherian rings. We need:

Lemma 6.6. Let $(A, \mathfrak{m})$ be a pair, and put $S=1+\mathfrak{m}, B=S^{-1} A, \mathfrak{n}=\mathfrak{m} B$. Then the canonical morphism $(A, \mathfrak{m}) \rightarrow(B, \mathfrak{n})$ induces an isomorphism $\left({ }^{h} A,{ }^{h} \mathfrak{m}\right) \sim\left({ }^{h} B,{ }^{h} \mathfrak{n}\right)$.

Proof. It is easy to see that the $N$-extensions of $(A, \mathfrak{m})$ and $(B, \mathfrak{n})$ are the same. The conclusion follows by Theorem 6.1(ii).

Corollary 6.7. Let $\phi:(A, \mathfrak{m}) \rightarrow\left({ }^{h} A,{ }^{h} \mathfrak{m}\right)$ be the canonical morphism. Then $\operatorname{Ker} \phi=\{x \in A \mid \exists m \in \mathfrak{m}$ such that $x=m x\}$.

Proof. With the notations of Lemma 6.6 we have $\mathfrak{n} \subset \operatorname{rad} B$. Hence ${ }^{h} B$ is faithfully flat over $B$ (Theorem 6.5 (ii)), whence $B \subset{ }^{h} B$. Therefore $\operatorname{Ker} \phi$ coincides with the kernel of the canonical homomorphism $A \rightarrow B$, whence the conclusion.

TheOREM 6.8. Let $(A, \mathfrak{m})$ be a pair and put $S=1+\mathfrak{n}$ and $B=S^{-1} A$. Then ${ }^{h} A$ is noetherian if and only if $B$ is noetherian. Moreover if this is the case $B$ and ${ }^{h} A$ have the same dimension.

Proof. By Lemma 6.6 and Theorem $6.5^{h} A$ is faithfully flat over $B$, whence if ${ }^{h} A$ is noetherian so is $B$ [2, p. 50, Corollaire]. Conversely suppose $B$ is noetherian and let $\mathfrak{n}=\mathfrak{m} B$. By Theorem 5.10 and Lemma 6.6 we have $\left({ }^{n} B,{ }^{n} \mathfrak{n}\right) \cong\left({ }^{n} B,{ }^{n} \mathfrak{n}\right)$ $\cong\left({ }^{h} A,{ }^{h} \mathfrak{m}\right)$. But ${ }^{n} B$ is noetherian by Proposition 5.5 (i), and thus ${ }^{h} A$ is noetherian. The last assertion follows by Theorem 6.1 (iv), since if $C$ is a Zariski ring and $\hat{C}$ is its completion, one has $\operatorname{dim} C=\operatorname{dim} \hat{C}$.

By Theorem 6.8 we have immediately:

Corollary 6.9. Let $\left({ }^{h} A,{ }^{h} \mathfrak{m}\right)$ be the H-closure of $(A, \mathfrak{m})$. Then

(i) If $A$ is noetherian, ${ }^{n} A$ is noetherian.

(ii) If ${ }^{h} A$ is noetherian and $\mathfrak{m} \subset \operatorname{rad} A$, then $A$ is noetherian.

To conclude this section we give another characterization of the henselization of a Zariski pair.

Definition 6.10. Let $(A, \mathfrak{m})$ be a subpair of the $H$-pair $(B, \mathfrak{n})$. The $H$-closure of $(A, \mathfrak{m})$ in $(B, \mathfrak{n})$ is the smallest $H$-pair contained in $(B, \mathfrak{n})$ and containing $(A, \mathfrak{m})$. 
It in easy to show that this "relative $H$-closure" exists, and is, in fact, the intersection of all $H$-pairs contained in $(B, \mathfrak{n})$ and containing $(A, \mathfrak{m})$. (For better details see $[10, \S 2]$.)

THEOREM 6.11. Let $(A, \mathfrak{m})$ be a Zariski pair (i.e. $A$ is noetherian and $\mathfrak{m} \subset \operatorname{rad} A$ ). Then the henselization of $(A, \mathfrak{m})$ is canonically isomorphic with the H-closure of $(A, \mathfrak{m})$ in $(\hat{A}, \hat{\mathfrak{m}})$.

Proof. Recall first that $(\hat{A}, \hat{\mathfrak{m}})$ is an $H$-pair $[3$, p. 88, Theorem 1], whence the $H$-closure of $(A, \mathfrak{m})$ in $(\hat{A}, \hat{\mathrm{m}})$ exists. Let us denote it by $(B, \mathfrak{n})$.

By Theorem 6.8 (i) $\left({ }^{n} A,{ }^{n} \mathfrak{m}\right)$ is a Zariski $H$-pair, and thus it is contained in its completion, which coincides with $(\hat{A}, \hat{\mathrm{m}})$ (Theorem 6.1 (iv)). Therefore by definition of $(B, \mathfrak{n})$ we have $(B, \mathfrak{n}) \subset\left({ }^{h} A,{ }^{h} \mathfrak{m}\right)$.

Moreover by the universal property of the $H$-closure (Definition 1.7) there is a unique morphism $\psi:\left({ }^{h} A,{ }^{h} \mathfrak{m}\right) \rightarrow(B, \mathfrak{n})$ such that the following diagram is commutative:

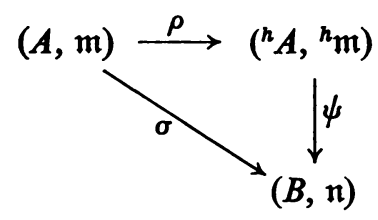

where $\rho$ and $\sigma$ are the immersions. Since $\left(\psi\left({ }^{n} A\right), \psi\left({ }^{n} \mathfrak{m}\right)\right)$ is an $H$-pair [7, Theorem 4.6] containing $(A, \mathfrak{m})$, we have that $\psi$ is onto, by definition of $(B, \mathfrak{n})$.

Finally if $\tau:(B, \mathfrak{n}) \rightarrow\left({ }^{h} A,{ }^{h} \mathfrak{m}\right)$ is the immersion, we have $\tau \circ \psi=\mathrm{id}$ (Definition $1.7)$, whence $\psi$ is also injective, and the proof is complete.

7. In this section we apply Theorem 6.1 to the study of some local properties of henselization. The main result we get (Theorem 7.4) is similar to a well-known theorem on $m$-adic completions of noetherian rings [3, p. 70, Proposition 8], and can be applied to show that several local properties of a ring (such as: locally normal, Cohen-Macaulay, $R_{k}, S_{k}$ ) are preserved by henselization (Corollaries 7.6, 7.7). We conclude this section with a sufficient condition for the henselization to be locally a domain (Corollary 7.9).

Since local rings can be regarded as particular pairs, it is clear what we intend by $N$-extension of a local ring. With this notion we have:

Lemma 7.1. Let $(B, \mathfrak{n})$ be an $N$-extension of $(A, \mathfrak{m})$ (Definition 2.2), and let $\mathfrak{p}$ be a maximal ideal of $A$ containing $\mathfrak{m}$. Then

(i) $\mathfrak{p} B$ is a maximal ideal of $B$.

(ii) $B_{\mathfrak{p}_{B}}$ is an $N$-extension of $A_{\mathfrak{p}}$.

Proof. Since the canonical morphism $(A, \mathfrak{m}) \rightarrow(B, \mathfrak{n})$ is strict (Proposition 2.6), we have immediately (i); as for (ii), it follows by a straightforward computation. 
REMARK 7.2. It is possible to show that, in Lemma 7:1, $\boldsymbol{B}_{\mathfrak{p}_{B}}$ is actually a simple $N$-extension of $A_{\mathfrak{p}}$ (Definition 2.2), as follows by [10, Proposition 6]. However Lemma 7.1 will be enough to us.

LEMMA 7.3. Let $(A, \mathfrak{m})$ be a pair, $\left(A_{\mathfrak{i}}, \mathfrak{m}_{\mathfrak{i}}\right)_{i \in I}$ a direct set of $N$-extension of $(A, \mathfrak{m})$, and $(B, \mathfrak{n})=\operatorname{inj} \lim \left(A_{\mathfrak{i}}, \mathfrak{m}_{\mathfrak{i}}\right)$. Then $\left({ }^{h} A,{ }^{n} \mathfrak{m}\right) \cong\left({ }^{h} B,{ }^{h} \mathfrak{n}\right)$.

Proof. Let $\left(A^{\prime}, \mathfrak{m}^{\prime}\right)$ be an $N$-extension of $(A, \mathfrak{m})$. Then every $N$-extension of $\left(A^{\prime}, \mathfrak{m}^{\prime}\right)$ is an $N$-extension of $(A, \mathfrak{m})$, and hence $\left({ }^{h} A^{\prime},{ }^{h} \mathfrak{m}^{\prime}\right) \cong\left({ }^{h} A,{ }^{h} \mathfrak{m}\right)$ (Theorem 6.1 (ii)).

The conclusion follows immediately since henselization commutes with direct limits [Corollary 6.3].

THEOREM 7.4. Let $(B, \mathfrak{n})$ be the henselization of $(A, \mathfrak{m})$. Then

(i) The map $\mathfrak{p} \rightarrow \mathfrak{p} B$ is a bijection of the set of maximal ideals of $A$ containing $\mathfrak{m}$, onto the set of all maximal ideals of $B$. Its inverse is the contraction.

(ii) If $\mathfrak{p}$ is a maximal ideal of $A$ containing $\mathfrak{m}$, then $A_{\mathfrak{p}}$ is canonically isomorphic to a subring of $B_{\mathfrak{p}_{B}}$ and $B_{\mathfrak{p}_{B}}$ is faithfully flat over $A$.

(iii) For every maximal ideal $\mathfrak{p}$ of $A$ containing $\mathfrak{m}$, the local rings $A_{\mathfrak{p}}$ and $B_{\mathfrak{p} B}$ have the same henselization and the same completion.

Proof. Since the canonical morphism $(A, \mathfrak{m}) \rightarrow(B, \mathfrak{n})$ is strict (Theorem 6.1 (iii)), it is easy to show (i). By Theorem 6.5 (i) and some well-known facts about flatness [2, p. 116, Proposition 15, and p. 44, Proposition 1] we have (ii).

By Theorem 6.1 (ii) we have $(B, \mathfrak{n})=\operatorname{inj} \lim \left(A_{\mathfrak{i}}, \mathfrak{m}_{\mathfrak{i}}\right)$, where $\left(A_{\mathfrak{i}}, \mathfrak{m}_{\mathfrak{i}}\right)_{\mathfrak{i} \in I}$ is the direct set of all $N$-extensions of $(A, \mathfrak{m})$. Let $\mathfrak{p}$ be a maximal ideal of $A$, containing $\mathfrak{m}$, and let $\mathfrak{p}_{i}=\mathfrak{p} A_{i}$ for every $i \in I$. Then (Lemma 7.1) $\mathfrak{p}_{i}$ is maximal for every $i$, and $\left(A_{\mathfrak{i}}\right)_{\mathfrak{p}_{\mathfrak{t}}}$ is an $N$-extension of $A_{\mathfrak{p}}$. It is easy to see that

$$
B_{\mathfrak{p}_{B}}=\operatorname{inj} \lim \left(A_{\mathfrak{i}}\right)_{\mathfrak{p}_{\mathfrak{t}}}
$$

whence (iii) follows by Lemma 7.3, and Theorem 6.1 (iv).

By Theorem 7.4 we get immediately

COROLlaRY 7.5. Let $P$ be a property of local rings such that either:

(a) A local ring has property $P$ if and only if its completion does; or

(b) A local ring has property $P$ if and only if its henselization does.

Let $\left({ }^{h} A,{ }^{n} \mathfrak{m}\right)$ be the henselization of $(A, \mathfrak{m})$. Then the following conditions are equivalent:

(i) $\left({ }^{h} A\right)_{\mathfrak{p}^{\prime}}$, has property $P$ for any maximal ideal $\mathfrak{p}^{\prime}$ of ${ }^{h} A$.

(ii) $A_{\mathfrak{p}}$ has property $P$ for any maximal ideal $\mathfrak{p}$ containing $\mathfrak{m}$.

Now we give a number of applications of Corollary 7.5.

COROLlary 7.6. Let $\left({ }^{n} A,{ }^{n} \mathfrak{m}\right)$ be the henselization of $(A, \mathfrak{m})$. Then the following conditions are equivalent:

(i) ${ }^{h} A$ is locally normal.

(ii) $A_{\mathfrak{p}}$ is normal for every maximal ideal $\mathfrak{p}$ containing $\mathfrak{m}$. 
Proof. Since a local ring is normal if and only if its henselization is normal [9, p. 139, Theorem 18.6.9 (i)], the conclusion follows immediately by Corollary 7.5.

We recall that a noetherian $\operatorname{ring} A$ is $R_{k}$ if $A_{\mathfrak{p}}$ is regular whenever $\mathfrak{p}$ is a prime ideal of height $k$; and that $A$ is $S_{k}$ if depth $A_{\mathfrak{p}} \geqq \min \{k, h t(\mathfrak{p})\}$. Thus a ring is regular if and only if it is $R_{k}$ for every $k$, and is Cohen-Macaulay if and only if it is $S_{k}$ for every $k$.

With this terminology we have:

Corollary 7.7. Let $\left({ }^{h} A,{ }^{h} \mathfrak{m}\right)$ be the henselization of $(A, \mathfrak{m})$ and let $P$ be any one of the following properties: $R_{k}, S_{k}$, regular, Cohen-Macaulay. Then

(i) If $A$ is $P$ so is ${ }^{h} A$.

(ii) If ${ }^{h} A$ is $P$ and $\mathfrak{m} \subset \operatorname{rad} A, A$ is $P$.

Proof. It is known that a local ring is $P$ if and only if its henselization is $P$ $[9$, p. 140 , Corollary 18.6.10]. Therefore the conclusion follows by Corollary 7.5 and Theorem 6.8 .

To conclude we give a criterion for local integrality. We recall first the following

Definition 7.8. [8, p. 217, 23.2.1] A local ring $A$ is unibranch if

(i) $A_{\text {red }}$ is a domain, and

(ii) the integral closure of $A_{\text {red }}$ is a local ring.

A ring $B$ is locally unibranch if $B_{\mathfrak{p}}$ is unibranch for every maximal ideal $\mathfrak{p}$.

Corollary 7.9. Let $\left({ }^{h} A,{ }^{h} \mathfrak{m}\right)$ be the henselization of $(A, \mathfrak{m})$. If $A_{\mathfrak{p}}$ is unibranch and reduced for every maximal ideal $\mathfrak{p}$ containing $\mathfrak{m}$, then ${ }^{h} A$ is locally a domain.

Proof. If a local ring is reduced and unibranch, its henselization is a domain [9, p. 141, Proposition 18.6.2], and the conclusion follows by Theorem 7.4.

8. The aim of this section is to prove that if $(B, \mathfrak{n})$ is the henselization of $(A, \mathfrak{m})$, then $\left(B_{\text {red }}, \mathfrak{n} B_{\text {red }}\right)$ is the henselization of $\left(A_{\text {red }}, \mathfrak{m} A_{\text {red }}\right)$ (Theorem 8.7). To obtain this result we study the behavior of the radical of an extended ideal (Theorem 8.3) and show that henselization depends essentially on $\sqrt{ } \mathfrak{m}$ rather than on $\mathfrak{m}$ (Proposition 8.6). To prove these results we make use mainly of Theorems 6.5 and 7.4. All the results obtained here are well known for henselization of local rings, but they are false, in general, for $\mathfrak{m}$-adic completions.

Lemma 8.1. Let $A, B$ be two local rings such that $B$ dominates $A$, and ${ }^{h} A={ }^{h} B$. Let $\mathfrak{a}$ be an ideal of $A$. Then $\mathfrak{a}=\sqrt{ } \mathfrak{a} \Rightarrow \mathfrak{a} B=\sqrt{ }(\mathfrak{a} B)$.

Proof. Let $A^{\prime}=A / \mathfrak{a}, B^{\prime}=B / \mathfrak{a} B$. Then $B^{\prime}$ is a local ring dominating $A^{\prime}$, and ${ }^{h} A^{\prime}={ }^{h} B^{\prime}$ [9, p. 139, Proposition 18.6.8]. Moreover $A^{\prime}$ is reduced, whence ${ }^{h} A^{\prime}$ and ${ }^{n} B^{\prime}$ are reduced $[9$, p. 139 , Theorem 18.6.9]. Thus $\mathfrak{a} B=\sqrt{ }(\mathfrak{a} B)$, and the proof is complete.

LemMA 8.2. Let $(B, \mathfrak{n})$ be the henselization of $(A, \mathfrak{m})$, and let $\mathfrak{a}$ be an ideal of $A$ such that $\mathfrak{a}=\sqrt{ } \mathfrak{a}$. Then $\mathfrak{a} B=\sqrt{ }(\mathfrak{a} B)$. 
Proof. It is sufficient to show that, for every maximal ideal $\mathfrak{p}$ of $B$, one has $\mathfrak{a} B_{\mathfrak{p}}=(\sqrt{ } \mathfrak{a}) B_{\mathfrak{p}}$. Now if $\mathfrak{q}$ is the contraction of $\mathfrak{p}$ to $A, \mathfrak{q}$ is maximal and $\mathfrak{p}=\mathfrak{q} B$ (Theorem 7.4 (i)), and moreover $A_{\mathfrak{q}}$ and $B_{\mathfrak{p}}$ have the same henselization (Theorem 7.4 (ii)). Since $\mathfrak{a} A_{\mathfrak{q}}=(v \mathfrak{a}) A_{\mathfrak{q}}=\sqrt{ }\left(\mathfrak{a} A_{\mathfrak{q}}\right)$, the conclusion follows easily by Lemma 8.1.

THEOREM 8.3. Let $(B, \mathfrak{n})$ be the henselization of $(A, \mathfrak{m})$, and let $\mathfrak{a}$ be an ideal of $A$. Then $\sqrt{ }(\mathfrak{a} B)=(\sqrt{ } \mathfrak{a}) B$.

Proof. It is clear that $\sqrt{ }(\mathfrak{a} B) \supset(\sqrt{ } \mathfrak{a}) B$. Moreover, since $\mathfrak{a} B \subset(\sqrt{ } \mathfrak{a}) B$ we have, by Lemma 8.2,

$$
\sqrt{ }(\mathfrak{a} B) \subset \sqrt{ }((\sqrt{ } \mathfrak{a}) B)=(\sqrt{ } \mathfrak{a}) B
$$

whence the conclusion.

If we apply Theorem 8.3 to the zero ideal of $A$ we get immediately:

COROLlary 8.4. Let $(B, \mathfrak{n})$ be the henselization of $(A, \mathfrak{m})$, and let $\mathfrak{a}$ be the nilradical of $A$. Then $\mathfrak{a} B$ is the nilradical of $B$.

Corollary 8.5. Let $(B, \mathfrak{n})$ be the henselization of $(A, \mathfrak{m})$. Let $S=1+\mathfrak{m}$ and $C=S^{-1} A$. Then the following conditions are equivalent:

(i) $B$ is reduced.

(ii) $C$ is reduced.

(iii) Every nilpotent of $A$ is annihilated by an element of $S$.

Proof. Since $(B, \mathfrak{n})=\left({ }^{h} C,{ }^{n}(\mathfrak{m} C)\right)\left(\right.$ Lemma 6.6) and $C \subset{ }^{h} C$ (Corollary 6.7) the conclusion follows easily by Corollary 8.4 .

Now we want to show that ${ }^{h}\left(A_{\text {red }}\right)=\left({ }^{h} A\right)_{\text {red }}$. In order to do this we need the following:

Proposition 8.6. Let $\left({ }^{h} A,{ }^{h} \mathfrak{m}\right)$ be the henselization of $(A, \mathfrak{m})$, and let $(B, \mathfrak{n})$ be the henselization of $(A, \sqrt{ } \mathfrak{m})$. Then we have $\left({ }^{h} A,{ }^{h} \mathfrak{m}\right)=(B, \mathfrak{m} B)$, and $\mathfrak{n}=\sqrt{ }(\mathfrak{m} B)$.

Proof. By Lemma 6.6 we may assume $\mathfrak{m} \subset \operatorname{rad} A$. Thus $B$ is faithfully flat over $A$ (Theorem 6.5(ii)), and $\mathfrak{m} B \cap A=\mathfrak{m}$. [2, p. 51, Proposition 9]. It follows that the canonical morphism $\psi: A \rightarrow B$ defines a morphism $\psi:(A, \mathfrak{m}) \rightarrow(B, \mathfrak{m} B)$.

Since $\mathfrak{m} B \subset \mathfrak{n}$, we have that $(B, \mathfrak{m} B)$ is an $H$-pair [7, Corollary 4.2] and, according to Definition 1.7, it remains to prove the following: for any $H$-pair $(R, \mathfrak{a})$ and any morphism $\rho:(A, \mathfrak{m}) \rightarrow(R, \mathfrak{a})$ there is a unique morphism ${ }^{n} \rho:(B, \mathfrak{m} B) \rightarrow(R, \mathfrak{a})$ such that the following diagram is commutative:

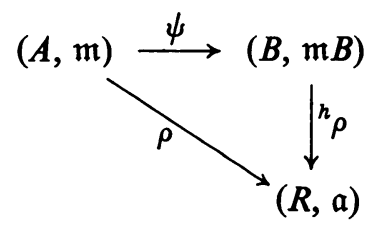


Recall that $(R, \mathfrak{a})$ is an $H$-pair if and only if $(R, \sqrt{ } \mathfrak{a})$ is an $H$-pair (see e.g. Corollary 4.7 and Theorem 5.11). Since $\rho^{-1}(\sqrt{ } \mathfrak{a})=\sqrt{ }\left(\rho^{-1}(\mathfrak{a})\right)=\sqrt{ } \mathfrak{m}, \rho$ is also a morphism $(A, \sqrt{ } \mathfrak{m}) \rightarrow(R, \sqrt{ } \mathfrak{a})$, and since $(R, \sqrt{ } \mathfrak{a})$ is an $H$-pair, there is a unique morphism ${ }^{n} \rho:(B, \mathfrak{n}) \rightarrow(R, \sqrt{ } \mathfrak{a})$ such that the following diagram is commutative:

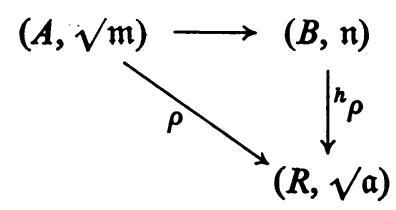

But it is easy to show that $\left({ }^{h} \rho\right)^{-1}(\mathfrak{a})=\mathfrak{m} B$, whence diagram (1) makes sense and is commutative. Since ${ }^{h} \rho$ is clearly unique, we have $\left({ }^{h} A,{ }^{n} \mathfrak{m}\right)=(B, \mathfrak{m} B)$. The last assertion follows then by Theorem 8.3 , and the proof is complete.

TheOREM 8.7. Let $(B, \mathfrak{n})$ be the henselization of $(A, \mathfrak{m})$, and let $C=A_{\text {red. }}$ Then $\left({ }^{n} C,{ }^{n}(\mathfrak{m} C)\right)=\left(B_{\text {red }}, \mathfrak{n} B_{\text {red }}\right)$.

Proof. Let $\mathfrak{a}$ be the nilradical of $A$, and suppose first that $\mathfrak{m}=\sqrt{ } \mathfrak{m}$. Then $\mathfrak{a} \subset \mathfrak{m}$, and by Corollary 6.4 we have $\left({ }^{h} C,{ }^{n}(\mathfrak{m} C)\right)=(B / \mathfrak{a} B, \mathfrak{m}(B / \mathfrak{a} B))$. But $\mathfrak{a} B$ is the nilradical of $B$ (Corollary 8.4 ) and the conclusion follows in this case. The general case follows easily by the previous one and Proposition 8.6.

9. We have seen (Theorem 6.8) that the henselization of a noetherian ring is noetherian. This fact, together with the local results obtained in $\$ 7$, will enable us to give criteria for the henselization of a noetherian ring to be a domain (Theorem 9.2), a normal ring (Theorem 9.5), a ring with irreducible spectrum (Theorem 9.7), and a UFD (Theorem 9.11). These results are similar to others known for $\mathfrak{m}$-adic completions (see e.g. [1] and [4]).

We begin with criteria of integrality. For this we need:

LeMmA 9.1. Let $\left({ }^{h} A,{ }^{h} \mathfrak{m}\right)$ be the henselization of $(A, \mathfrak{m})$. Then $\operatorname{Spec}(A / \mathfrak{m})$ is connected if and only if $\operatorname{spec}\left({ }^{h} A\right)$ is connected.

Proof. By Theorem 6.1 (iii) we have $A / \mathfrak{m} \cong{ }^{h} A /{ }^{h} \mathfrak{m}$, and since $\left({ }^{h} A,{ }^{h} \mathfrak{m}\right)$ is an $H$ pair, the conclusion follows by [7, Corollary 1.9].

THEOREM 9.2. Let $A$ be a locally unibranch ring (Definition 7.8), and $\mathfrak{m} \subset \operatorname{rad} A$ an ideal of $A$. Let $\left({ }^{n} A,{ }^{h} \mathfrak{m}\right)$ be the henselization of $(A, \mathfrak{m})$. Then the following conditions are equivalent:

(i) ${ }^{n} A$ is a noetherian domain.

(ii) $A$ is a noetherian domain and $\operatorname{spec}(A / \mathfrak{m})$ is connected.

(iii) $A$ is noetherian and reduced and $\operatorname{spec}(A / \mathfrak{m})$ is connected. 
Proof. Suppose ${ }^{h} A$ is a noetherian domain. Then $A$ is a noetherian domain by Theorem 6.7 (ii), and $\operatorname{spec}(A / \mathrm{m})$ is connected by Lemma 7.1. Thus (i) $\Rightarrow$ (ii). It is clear that (ii) $\Rightarrow$ (iii).

Suppose now (iii) is true. Then ${ }^{h} A$ is noetherian (Theorem 6.8) and is locally a domain (Corollary 7.9). Moreover $\operatorname{spec}\left({ }^{h} A\right)$ is connected (Lemma 9.1), and then ${ }^{n} A$ is a domain (see e.g. [4], Proposition 2.6).

By Theorem 9.2 and Lemma 6.6 we get easily

COROLlaRY 9.3. Let $\left({ }^{n} A,{ }^{n} \mathfrak{m}\right)$ be the henselization of $(A, \mathfrak{m})$, and suppose

(i) $A$ is noetherian and reduced,

(ii) $A_{\mathfrak{p}}$ is unibranch for every maximal ideal $\mathfrak{p}$ containing $\mathfrak{m}$.

Then ${ }^{h} A$ is a domain if and only if $\operatorname{spec}(A / \mathfrak{m})$ is connected.

Now we will study irreducibility of $\operatorname{spec}\left({ }^{n} A\right)$. We recall first

Lemma 9.4. Let $C$ be a ring. Then the following conditions are equivalent:

(i) Spec $(C)$ is connected.

(ii) Spec $(C / \mathfrak{a})$ is connected for every nilideal a of $C$,

(iii) there is a nilideal $\mathfrak{a}$ of $C$ such that $\operatorname{spec}(C / \mathfrak{a})$ is connected.

Proof. If $\mathfrak{a}$ is a nilideal of $C$ the canonical map $\operatorname{spec}(C / \mathfrak{a}) \rightarrow \operatorname{spec}(C)$ is a homeomorphism, and the conclusion follows.

THEOREM 9.5. Let $\left({ }^{h} A,{ }^{h} \mathfrak{m}\right)$ be the henselization of $(A, \mathfrak{m})$. Suppose that $A$ is a noetherian locally unibranch ring, and that $\mathfrak{m} \subset \operatorname{rad} A$. Then the following conditions are equivalent:

(i) $\operatorname{spec}\left({ }^{h} A\right)$ is irreducible.

(ii) $\operatorname{spec}(A)$ is irreducible and $\operatorname{spec}(A / \mathfrak{m})$ is connected.

Proof. Let $B=A_{\text {red }}, \mathfrak{n}=\mathfrak{m} B$ and $\left({ }^{n} B,{ }^{n} \mathfrak{n}\right)$ the henselization of $(B, \mathfrak{n})$. By Theorem 8.7 we have ${ }^{h} B=\left({ }^{h} A\right)_{\text {red }}$. Observe further that $B$ is locally unibranch: this allows us to use Theorem 9.3.

Suppose now (i) holds. This means that $\left({ }^{h} A\right)_{\text {red }}$ is a domain, [2, p. 129, Corollary 1], and hence $B=A_{\text {red }}$ is a domain and $\operatorname{spec}(B / \mathfrak{n})$ is connected (Theorem 9.3). It follows immediately that $\operatorname{spec}(A)$ is irreducible; moreover $B / \sqrt{ } \mathfrak{n}=A / \sqrt{ } \mathfrak{m}$, and then $\operatorname{spec}(A / \mathfrak{m})$ is connected by Lemma 9.4 . Thus (i) $\Rightarrow$ (ii).

The converse is proved in a similar way by using Theorem 9.3 again.

By Theorem 9.5 and Lemma 6.6 we have easily

Corollary 9.6. Let $\left({ }^{h} A,{ }^{h} \mathfrak{m}\right)$ be the henselization of $(A, \mathfrak{m})$. Suppose $A$ is noetherian and $A_{\mathfrak{p}}$ is unibranch for every maximal ideal $\mathfrak{p}$ containing $\mathfrak{m}$. Then if $\operatorname{spec}(A)$ is irreducible, $\operatorname{spec}\left({ }^{h} A\right)$ is irreducible.

Now we give a criterion for the henselization to be normal. 
THEOREM 9.7. Let $\left({ }^{h} A,{ }^{h} \mathfrak{m}\right)$ be the henselization of $(A, \mathfrak{m})$, and suppose $A$ is noetherian. Then the following conditions are equivalent:

(i) ${ }^{n} A$ is normal.

(ii) Spec $(A / \mathfrak{m})$ is connected, and $A_{\mathfrak{p}}$ is normal for every maximal ideal $\mathfrak{p}$ containing $\mathfrak{m}$.

Proof. Suppose ${ }^{h} A$ is normal. Then $\operatorname{spec}(A / \mathfrak{m})$ is connected by Lemma 9.1 , and $A_{\mathfrak{p}}$ is normal for every maximal ideal $\mathfrak{p}$ containing $\mathfrak{m}$ (Corollary 7.6 ). Thus (i) $\Rightarrow$ (ii).

Conversely, if (ii) is true, ${ }^{n} A$ is a domain by Theorem 9.3 , and is locally normal (Corollary 7.6), whence ${ }^{h} A$ is normal. Thus (ii) $\Rightarrow$ (i) and the proof is complete.

To conclude this section we point out some relations between unique factorization of $A$ and ${ }^{h} A$. We recall first:

Theorem 9.8 [1, TheORem 3.10]. Let $(A, \mathfrak{m}) \subset(B, \mathfrak{n})$ be two Zariski pairs such that $(\hat{A}, \hat{\mathfrak{m}})=(\hat{B}, \hat{\mathfrak{n}})$. Then if $B$ is a UFD, $A$ is a UFD.

Corollary 9.9. Let $(B, \mathfrak{n})$ be the henselization of $(A, \mathfrak{m})$, and suppose $\mathfrak{m} \subset \operatorname{rad} A$. Then if $B$ is a noetherian UFD, $A$ is a noetherian UFD.

Proof. $A$ is noetherian by Theorem 6.7. Moreover $(\hat{B}, \hat{\mathfrak{n}}) \cong(\hat{A}, \hat{\mathfrak{m}})$, and since $\mathfrak{n} \subset \operatorname{rad} B$ (Definition 1.1), the conclusion follows by Theorem 9.8.

By Corollary 9.9 and Theorem 7.4 (iii) we have

COROLlaRY 9.10. Let $(B, \mathfrak{n})$ be the henselization of $(A, \mathfrak{m})$, and suppose that for every maximal ideal $\mathfrak{p}$ of $A$ containing $\mathfrak{m}$, the henselization of the local ring $A_{\mathfrak{p}}$ is a noetherian UFD. Then $B$ is locally UFD.

We give now a criterion for the factoriality of the henselization.

THEOREM 9.11. Let $\left({ }^{h} A,{ }^{h} \mathfrak{m}\right)$ be the henselization of $(A, \mathfrak{m})$. Suppose $A$ is noetherian, and $\left({ }^{n} A\right)_{\mathfrak{p}}$ is a UFD for every maximal ideal $\mathfrak{p}$ containing $\mathfrak{m}$. Then the following conditions are equivalent:

(i) ${ }^{n} A$ is a UFD.

(ii) $\operatorname{Spec}(A / \mathfrak{m})$ is connected, and $P(A / \mathfrak{m})=0$ (for the definition of $P$ see $[2, \mathrm{p} .144])$.

Proof. If $(B, \mathfrak{n})$ is an $H$-pair, the canonical homomorphism $P(B) \rightarrow P(B / \mathfrak{n})$ is bijective [7, Corollary 5.5], and since $A / \mathfrak{m}={ }^{n} A /{ }^{h} \mathfrak{m}$ (Theorem 6.1) we have $P\left({ }^{h} A\right.$ ) $\cong P(A / \mathfrak{m})$.

Suppose now ${ }^{h} A$ is a UFD. Then $P\left({ }^{h} A\right)=0$, whence $P(A / \mathfrak{m})=0$. Moreover ${ }^{h} A$ is a domain, whence $\operatorname{spec}(A / \mathfrak{m})$ is connected. Thus (i) $\Rightarrow$ (ii).

Conversely, if (ii) holds, ${ }^{h} A$ is a domain by Theorem 9.3 , and is locally UFD by Corollary 9.10. By the above remark we have also $P\left({ }^{n} A\right)=0$, and is then easy to see that ${ }^{h} A$ is UFD. (For better details see e.g. [4].)

Since a regular ring is locally UFD, by Corollary 7.7 and the above theorem we have 
Corollary 9.12. Let $\left({ }^{h} A,{ }^{h} \mathfrak{m}\right)$ be the henselization of $(A, \mathfrak{m})$, and suppose $A$ is regular. Then ${ }^{h} A$ is a UFD if and only if $P(A / \mathfrak{m})=0$ and $\operatorname{Spec}(A / \mathfrak{m})$ is connected.

REMARK 9.13. The proofs given in this section are quite similar to analog ones used in [4] to study m-adic completion. For this reason we have supposed the rings were noetherian. It is however quite possible that this hypothesis could be much weakened, mainly by using those properties of henselization which are true without noetherian assumptions (as e.g. Theorem 6.5 and Corollary 7.5 etc.).

Added in proof. After sending the manuscript of the present paper $I$ found out that E. Crepaux had given a different proof of Theorem 5.11 (E. Crepaux, Une caractérisation des couples henseliennes, Enseignement Math. (2) 13 (1968), 273-279). Since our Theorem 5.11 follows immediately by Theorems 3.5 and 5.11, by using Crepaux's result one could avoid the whole $\S 4$ of the present paper (while the main part of $\$ 5$ is needed later). I prefer, however, to keep the actual form, since the two proofs of Theorem 5.10 are quite different, and the main result of $\$ 4$ (Proposition 4.10) was announced without proof [5, Theorem 2.6].

\section{REFERENCES}

1. M. Arezzo and S. Greco, Sul gruppo delle classi di ideali. Ann. Scuola Norm. Sup. Pisa 21 (1967), 459-483.

2. N. Bourbaki, Algèbre commutative. I, II, Hermann, Paris, 1961.

3. - Algèbre commutative. III, IV, Hermann, Paris, 1961.

4. S. Greco, Sull' integrità e la fattorialità dei completamenti m-adici, Rend. Sem. Mat. Univ. Padova 36 (1966), 50-65.

5. —-, Piattezza della chiusura henseliana, Rend. Accad. Naz. dei Lincei 43 (1967), 329-331.

6. - Qualche condizione di henselianità per anelli non locali, Le Matematiche (Catania) 22 (1967), 340-359.

7. — Algebras over nonlocal Hensel rings, J. Algebra 8 (1968), 45-59.

8. A. Grothendieck, Eléments de géométrie algébrique, Chapter IV, Part 1, Publ. Math. No. 20, Inst. Hautes Études Sci., 1960.

9. —_ Eléments de géométrie algébrique, Chapter IV, Part 4, Publ. Math. No. 32, Inst. Hautes Études Sci., 1968.

10. J. P. Lafon, Anneaux Henseliens, Bull. Soc. Math. France 91 (1963), 77-107.

11. M. Nagata, On the theory of henselian rings, Nagoya Math. J. 5 (1953), 45-57.

12. - On the theory of henselian rings. II, Nagoya Math. J. 7 (1954), 1-19.

13. —, Local rings, Interscience, New York, 1962.

Istituto Matematico,

Genova, Italy 